\title{
Prevalência de tabagismo em uma unidade de internação psiquiátrica de Porto Alegre
}

\author{
Raquel De Boni* \\ Flávio Pechansky*
}

\section{INTRODUÇÃO}

O tabagismo é responsável por $20 \%$ das mortes nos EUA, estando relacionado às principais causas de morte da população adulta: doenças cardiovasculares, câncer e doenças respiratórias $^{1,2}$. Atualmente existe 1 bilhão e 200 milhões de pessoas fumantes no mundo, sendo que sua prevalência na população adulta norte -americana é de $25 \%$, o que é aproximadamente igual a estudo realizado em São Paulo, que encontrou prevalência de $24 \%{ }^{1-3}$. Segundo estimativas do Instituto Nacional do Câncer (INCA), no Brasil ocorrem 200.000 mortes/ano associadas ao tabagismo, o que significa que 23 pessoas morrem a cada hora. ${ }^{3}$

Vários estudos têm demonstrado a comorbidade entre doenças psiquiátricas graves (como esquizofrenia e transtornos do humor) e dependência de nicotina ${ }^{4-7}$, sendo que alguns autores sugerem que pacientes psiquiátricos apresentam dependência mais intensa que fu-

\footnotetext{
* Departamento de Psiquiatria e Medicina Legal - Universidade Federal do Rio Grande do Sul, Porto Alegre, Brasil.

* Centro de Pesquisa em Álcool e Drogas UFRGS, Porto Alegre, Brasil.
}

mantes sem comorbidade ${ }^{6}$. Entretanto, muitas vezes este diagnóstico não é realizado nos pacientes de unidades psiquiátricas ${ }^{7}$, e os sintomas de abstinência de nicotina (ansiedade, irritabilidade, humor deprimido, insônia) podem ser confundidos com os sintomas que levaram à internação. O Serviço de Psiquiatria do HCPA recomendou a avaliação dos hábitos de fumo dos pacientes atendidos na internação psiquiátrica deste hospital, pelo desconforto relatado pelos pacientes internados em função da abstinência ao tabaco, dentro de um projeto de abordagem ao tabagismo que se faz de forma regular dentro da instituição.

O objetivo desta comunicação breve é descrever os achados iniciais de prevalência de tabagismo em pacientes internados na Unidade de Internação Psiquiátrica do Hospital de Clínicas de Porto Alegre.

\section{MÉTODO}

Foram realizados três censos consecutivos, com intervalo de trinta dias cada um, nos pacientes internados na Unidade Psiquiátrica do HCPA, cuja lotação máxima é de 36 pacien-

Recebido em 08/09/2003. Revisado em 07/10/2003. Aprovado em 04/11/2003. 
tes. Foram escolhidos trinta dias em virtude da rotatividade dos pacientes, em função da taxa média de permanência na unidade. Mediante consentimento verbal, todos responderam ao Questionário de Fagerström, que avalia a intensidade da dependência ao tabaco. O questionário foi elaborado em $1978{ }^{8}$ e é composto por seis perguntas com diferentes pesos, sendo que o escore máximo é 10 . O ponto de corte para identificar dependência grave ainda é controverso, tendo sido adotado neste estudo o ponto de corte de seis ou mais pontos. ${ }^{1,9,10}$. Até o presente momento, os autores não identificaram a validação concorrente do instrumento no Brasil. O questionário foi aplicado por estagiários de pesquisa treinados.

\section{RESULTADOS}

No primeiro censo foram entrevistados 32 pacientes, no segundo 35 e no terceiro, 36 pacientes. A porcentagem de fumantes encontrada foi de $46,7 \%$ no primeiro censo, de $54,3 \%$ no segundo e $38,9 \%$ no terceiro. No primeiro censo, $53,3 \%$ dos tabagistas eram mulheres; no segundo, $57,9 \%$ eram homens; e no terceiro, a proporção entre homens e mulheres foi idêntica. Em relação à idade, os entrevistados entre 19 e 30 anos foram maioria nos três momentos. As taxas de fumantes graves foram de $46,7 \%$ no primeiro censo, de $47,6 \%$ no segundo e $50 \%$ no terceiro. A pontuação média dos dependentes graves encontrada foi de 6,71 no primeiro censo; 7,44 no segundo e 7,57 no terceiro. Não houve diferença estatisticamente significativa entre as três amostras $(p>0,05)$. Os achados encontram-se descritos na Tabela 1.

Tabela 1. Comparação entre as prevalências de tabagismo nos três censos

\begin{tabular}{lllll}
\hline & $\begin{array}{l}\text { Censo 1 } \\
\mathrm{n}(\%)\end{array}$ & $\begin{array}{l}\text { Censo 2 } \\
\mathrm{n}(\%)\end{array}$ & $\begin{array}{l}\text { Censo 3 } \\
\mathrm{n}(\%)\end{array}$ & $\mathrm{p}$ \\
\hline Prevalência & $15(46,7)$ & $19(54,3)$ & $14(38,9)$ & $\mathrm{ns}$ \\
Homens & $7(46,7)$ & $11(57,9)$ & $7(50)$ & $\mathrm{ns}$ \\
Mulheres & $8(53,3)$ & $8(42,1)$ & $7(50)$ & \\
$\begin{array}{l}\text { Dependência } \\
\text { Grave }\end{array}$ & $7(46,7)$ & $9(47,6)$ & $3(50)$ & $\mathrm{ns}$ \\
\hline
\end{tabular}

$n s=$ não significativo $(p>0,05)$

\section{DISCUSSÃO}

Embora este seja um estudo de pequenas proporções, limitado a uma variável única e a confirmam uma alta prevalência de fumantes na Unidade de Internação Psiquiátrica (aproximadamente $50 \%$ dos pacientes internados), consideravelmente maior do que a encontrada na população geral e maior do que a encontrada em pacientes internados de um hospital geral paulista $(21 \%) .{ }^{9}$ Existe uma proporção significativa de dependentes graves em relação aos fumantes em menor grau. Tais achados vão ao encontro de dados descritos por outros autores em amostras semelhantes ${ }^{4-6}$. Ressalta-se que não houve diferença significativa entre as três amostras, sugerindo que os achados acima descritos apresentam constância na unidade de internação psiquiátrica estudada.

Além da gravidade da dependência, que pode estar diretamente relacionada com os sintomas de abstinência, vários estudos sugerem que pacientes com episódios prévios de depressão apresentam recorrência após a abstinência de nicotina ${ }^{11,12}$; mais ainda, que a nicotina pode diminuir a concentração sérica dos fármacos anti-psicóticos, o que têm implicações no tratamento dos pacientes em abstinência. ${ }^{13}$

Também foi observada uma prevalência alta em pacientes jovens (19-30 anos), o que aponta para a necessidade de prevenção do tabagismo em adolescentes, visto que a idade média de experimentação é de 13 anos. A nicotina irá levar a um uso problemático 30-60\% destes jovens, e apenas $2,5 \%$ dos pacientes dependentes irão alcançar a abstinência a cada ano, de acordo com o consenso brasileiro sobre o tratamento da dependência de nicotina. ${ }^{1}$

\section{CONCLUSÕES}

Os dados acima descritos justificam abordagens terapêuticas que visem a minimizar os sintomas de abstinência à nicotina e reforçam a importância de avaliar tal dependência nos pacientes psiquiátricos, particularmente no início de seu tramento hospitalar. O consenso brasileiro sobre o tratamento da dependência de nicotina ${ }^{1}$ recomenda a reposição de nicotina para a maioria dos pacientes, exceto grávidas e idosos com risco cardiovascular, para o tratamento da abstinência. Sua associação com terapia comportamental breve em grupo seria de fácil execução e custo aceitável ${ }^{1}$, podendo ser indicada para os pacientes motivados a manter a abstinência.

Ao considerar a amostra específica de pacientes psiquiátricos, não foi objetivo deste estudo avaliar comorbidades. Entretanto, cabe ressaltar a importância da realização de estu- 
dos que avaliem as comorbidades psiquiátricas em pacientes tabagistas brasileiros, visto que o tratamento dos transtornos associados pode ser um fator importante para a elevação das taxas de abstinência ao tabaco.

\section{AGRADECIMENTOS}

Os autores agradecem à colaboração do Dr. Iverson Brandão, pelo auxílio valioso na coleta dos dados.

\section{REFERÊNCIAS BIBLIOGRÁFICAS}

1. Marques ACPR Campana A, Gigliotti AP Lourenço MTC Ferreira MP, Laranjeira R. Consenso sobre o tratamento da dependência de nicotina. Rev Bras Psiquiatr 2001 23(4):200-14.

2. Fagerstrom K. The epidemiology of smoking: health consequences and benefits of cessation.Drugs. 2002;62 Suppl 2:1-9.

3. Ministério da Saúde. Secretaria de Assistência à Saúde. Instituto Nacional de Câncer - INCA. Estimativas da Incidência e Mortalidade por Câncer. Rio de Janeiro: INCA, 2002.

4. Itkin $\mathrm{O}$, Nemets B, Einat $\mathrm{H}$. Smoking habits in bipolar and schizophrenic outpatients in southern Israel.J Clin Psychiatry. $2001 \mathrm{Apr}$; 62(4):269-72.

5. de Leon J, Becona E, Gurpegui M, Gonzales-Pinto A Diaz FJ.The association between high nicotine dependence and severe mental illness may be consistent across countries. J Clin Psychiatry. 2002 Sep;63 (9):812-6.

6. Patkar AA, Gopalakrishnan R, Lundy A, Leone TT, Certa $\mathrm{KM}$, Weinstein SP. Relationship between tobacco smoking and positive and negative symptoms in schizophrenia. J Nerv Ment Dis.2002 Sep; 190(9):604-10.

7. Upadhyaya HP, Brady KT, Wharton M, Liao J. Psychiatric disorders and cigarette smoking among child and adolescent psychiatry inpatients. Am J Addict. 2003 Mar-Apr; 12(2):144-52.

8. Fagerstrom KO .Measuring degree of physical dependence to tobacco smoking with reference to individualization of treatment.Addict Behav. 1978; 3(3-4):235-41.

9. Figlie NB, Pillon SC, Dunn J, Laranjeira R. The frequency of smoking and problem drinking among general hospital inpatients in Brazil - using the AUDIT and Fagerstrom questionnaires.Sao Paulo Med J. 2000 Sep 7; 118(5):13943.

10. Halty LS, Huttener MD, Netto ICO, Santos VA, Martins G Análise da utilização do Questionário de Tolerância de Fargestron (QTF) como medida da dependência de nicotina. J. Pneumol 2002; 28(4):180-86.

11. Covey LS, Glassman AH, Stetner F. Major depression following smoking cessation.Am J Psychiatry 1997; 154:263-65.

12. Breslau n, Peterson EL, Schultz LR, Chilcoat HD, Andreski $P$. Major dpression and stages of smoking.Arch Gen Psychiatry 1998; 55:161-66.

13. Cordioli AV e cols. Psicofármacos- consulta rápida. 2 edição. ARTMED, Porto Alegre, 2000.

\section{RESUMO}

Em função de impressões clínicas sobre a alta prevalência de tabagismo nos pacientes psiquiátri- cos internados, o Serviço de Psiquiatria do HCPA solicitou um estudo que avaliasse a prevalência de tabagismo na Unidade de Internação Psiquiátrica deste hospital. Foram realizados três censos consecutivos, com intervalo de trinta dias, em pacientes internados na Unidade $(n=103)$. Foi utilizado o Questionário de Fagerström para mensurar a intensidade da dependência de nicotina. A prevalência de tabagismo foi de 46,7\%, 54, 3\% e 38,9\% no primeiro, segundo e terceiro censo, respectivamente ( $p>0,05)$. A dependência foi considerada grave em aproximadamente $50 \%$ dos tabagistas nos três censos. A alta prevalência de tabagismo e a maior gravidade da dependência em pacientes psiquiátricos confirmam dados da literatura e justificam intervenções com a finalidade de minimizar os sintomas de abstinência que podem ser confundidos com sintomas dos transtornos de base - em pacientes psiquiátricos internados.

Descritores: Tabagismo, prevalência, comorbidade.

\section{ABSTRACT}

Based on anecdotal clinical reports about the high prevalence of tobacco dependence in psychiatric inpatients, the Psychiatric Services of Hospital de Clinicas of Porto Alegre requested a study to ascertain its prevalence. Three consecutive censuses were developed, each 30 days apart, with a total of 103 in-patients, utilizing the Fagerström questionnaire for tobacco dependence to ascertain the severity of nicotine dependence. The prevalences found were $46.7 \%, 54.3 \%$ and $38.9 \%$, respectively, in the three consecutive samples $(p>0.05)$. Dependence was categorized as severe in approximately $50 \%$ of all smokers in the three censuses. The high prevalence of nicotine dependence and the highest severity of dependence among the psychiatric patients confirms literature data and justifies interventions aimed at minimizing withdrawal symptoms - which may be confounded with symptoms of the psychiatric primary diagnosis - in patients hospitalized for psychiatric disorders.

Key-words: Tobacco dependence, prevalence, comorbidity.

Title: Smoking prevalence in a Psychiatric Unit in Porto Alegre, Brazil

\section{RESUMEN}

En función de las impresiones clínicas sobre la alta predominancia de tabaquismo en los pacientes psiquiátricos internados, el servicio de Psiquiatría do HCPA solicito un estudio que evaluase la predominancia de tabaquismo en la Unidad de Internación Psiquiátrica de este Hospital. Fueron realizados tres 
censos consecutivos, con intervalo de treinta días, en pacientes internados en la Unidad $(n=103)$. Fue utilizado el Cuestionario de Fagestron para manejar la intensidad de la dependencia de nicotina. La predominancia de tabaquismo fue de $46.7 \%, 54.3 \%$ y $38.9 \%$ en el primer, segundo y tercer censo respectivamente $(p>0,05)$. La predominancia fue considerada grave en aproximadamente $50 \%$ de los tabaquistas en los tres censos. La alta predominancia de tabaquismo y la mayor gravedad de la dependencia en pacientes psiquiátricos confirman datos de la literatura y justifican intervenciones con la finalidad de minimizar los síntomas de abstinencia - que pueden ser confundidos con los síntomas de los trastornos de base - en pacientes psiquiátricos internados.
Palabras-clave: Tabaquismo, prevalência, comorbidad.

Título: Predominancia de tabaquismo en una unidad de internación Psiquiátrica de Porto Alegre

Endereço para correspondência:

Raquel De Boni

Centro de Pesquisa em Álcool e Drogas da UFRGS

Rua Itaqui 99/502

90460-140 - Porto Alegre - RS - Brasil

E-mail: raqueldeboni@msn.com

Copyright $\odot$ Revista de Psiquiatria do Rio Grande do Sul - SPRS 